\title{
Activos de propiedad intelectual y destinos turísticos inteligentes: una aproximación jurídica-empresarial
}

\author{
Dra. Sheila Sánchez Bergara \\ Ostelea School of Tourism and Hospitality, Barcelona, España \\ Departamento de Ciencias Sociales y Humanas \\ ssanchezb@ostelea.com
}

\section{Resumen}

Actualmente las tecnologías de la información y las comunicaciones son un componente clave de la actividad turística y cada vez más el uso que de ellas se hace incide en la competitividad de empresas y destinos. Este contexto impone desafíos en cuanto a los activos de propiedad intelectual que se usan y crean en el entorno digital. A través de un primer estudio cualitativo se profundiza en las cuestiones de propiedad intelectual que mayor relevancia tienen en el actual contexto tecnológico. Para ello se parte de un análisis de la literatura y del marco regulatorio de la propiedad intelectual vigente en España, se contrastan empíricamente las prácticas de un grupo de empresas del sector comprometidas con la innovación y se construyen una serie de inferencias teóricas aplicables tanto a nivel empresarial como de destino. Finalmente, se discuten los principales ámbitos de incidencia entre los activos de propiedad intelectual, las empresas y los DTI y se ofrecen un conjunto de recomendaciones.

Palabras claves: activos de propiedad intelectual, destinos turísticos inteligentes, gestión estratégica.

\section{Abstract}

Nowadays, ICTs have a key role in the frame of the tourist activity and the implementation of these technologies affects the competitiveness of companies and destinations' more and more. This is a challenging context for intellectual property assets used and created in the digital environment. A first qualitative study is proposed to deepen the main IP related issues in the current technological context. In this sense, the work starts with a literature review and the analysis of the IP regulatory framework in force in Spain. Then, the practices of a group of companies, particularly 
Actas del Seminario Internacional Destinos Turísticos Inteligentes:

nuevos horizontes en la investigación y gestión del turismo

Universidad de Alicante, 26 y 27 de octubre de 2017

committed with innovation are empirically contrasted and a range of theoretical inferences applicable at both company and destination level are highlighted. Finally, the main areas of interest among the intellectual property assets, companies and the DTIs are discussed and a range of recommendation are proposed.

\section{Introducción}

Para el desarrollo y gestión de un destino turístico inteligente (DTI) uno de los pilares básicos es la incorporación de las nuevas tecnologías de la información y las comunicaciones (NTIC). Sin embargo, el uso de las NTIC, concretamente las tecnologías digitales e Internet, por una parte, ofrece amplias posibilidades de usar y crear activos que pueden ser protegidos por la propiedad intelectual -ej. bases de datos, programas de ordenador, aplicaciones para móviles, obras multimedias, etc.-. Por otra parte, abre un nuevo escenario en el cual, gestionar estos activos deja de ser una cuestión meramente legal y se convierte en un factor estratégico incrustado en el núcleo de capacidades de la empresa (Smith \& Hansen, 2002). Además, la gestión de estos activos permite obtener el retorno del trabajo, el tiempo y los recursos invertidos (Spinello, 2007) y contribuye al desarrollo local y el bienestar social (Santagata, 2002, 2006).

No obstante, todavía son escasas las investigaciones enfocadas en conectar las cuestiones legales de los activos de propiedad intelectual con el turismo (Ghafele \& Santagata, 2006). Ni la literatura turística, ni la de gestión ha profundizado en la relación entre estas áreas, en buena medida porque el sector turístico tradicionalmente ha sido considerado en su rol de consumidor, es decir, únicamente como un valioso nicho de mercado (UNCTAD, 2010). Una revisión de la literatura permite constatar que respecto a la gestión de los activos de propiedad intelectual, los estudios empíricos centran su atención en las industrias tecnológicas, culturales y creativas (e.g. Garon, 2012; Kankanala, 2012 y Mukundan \& Jain, 2012). Por otra parte, desde el ámbito turístico los estudios han abordado, fundamentalmente, cuestiones legales relativas a las marcas y las denominaciones de origen (e.g. Ghafele \& Santagata, 2006; Russo, 2013; Segre \& Russo, 2005 y Russo \& Segre, 2009); siendo escasas las contribuciones relativas a los derechos de autor y el turismo (e.g. Ide, 2008). Sin embargo, algunas investigaciones han reconocido la necesidad de tener en cuenta estas cuestiones legales (e.g. Ohashi et al., 2012 y Rodríguez et al., 2012). 
Actas del Seminario Internacional Destinos Turísticos Inteligentes:

nuevos horizontes en la investigación y gestión del turismo

Universidad de Alicante, 26 y 27 de octubre de 2017

La literatura especializada en activos de propiedad intelectual concibe la gestión como un proceso que persigue la maximización económica de estos activos a través de una serie de fases, tales como: la generación, la valorización, la protección, la explotación y la vigilancia (Smith \& Hansen, 2002 y Punnoose \& Shobhana, 2012). Estas fases a su vez, forman parte de la práctica habitual de las empresas tradicionalmente creadoras -industrias culturales, creativas y tecnológicas- para gestionar su cartera de activos de propiedad intelectual. En este escenario, y a diferencia de la literatura previa, esta investigación analiza las prácticas de un grupo de empresas turísticas respecto a la gestión de activos de propiedad intelectual, el marco regulatorio vigente y sus incidencias en el funcionamiento de los DTI. Con este propósito se realiza una revisión de la literatura y las fuentes legales de mayor trascendencia, se incluyen datos de un trabajo empírico previo y se contrastan entre sí. Finalmente, se discuten unas conclusiones tanto a nivel de empresa como de destino y se ofrecen algunas recomendaciones generales para directivos y entidades del sector.

\section{Las NTIC y los destinos turísticos}

En las últimas décadas, la literatura ha abordado ampliamente los efectos de las NTIC en el turismo. El sector siempre ha sido un consumidor de información, no en balde, Sheldon (1997) sostenía que esta última era el alma del turismo y las tecnologías de la información y las comunicaciones su columna vertebral. Los avances tecnológicos en esta área han devenido en un elemento transversal que cobra especial relevancia para una gestión inteligente de los destinos turísticos. Por una parte, es un elemento clave para el almacenamiento y gestión de información básica para que los servicios, las infraestructuras y el patrimonio del destino funcionen de manera inteligente (SEGITTUR, 2015). Por otra parte, favorece los procesos de comunicación e interacción entre los distintos agentes (SEGITTUR, 2015), permitiendo la colaboración entre los mismos (Miralbell y Sivera, 2008), elemento que constituye un factor básico en los destinos 2.0 (William, 2010).

Las NTIC han permitido la digitalización de todos los procesos de la cadena de valor turística, según se constata de lo expresado por Buhalis (2003) respecto al e-tourism. Esta digitalización, a su vez, ha supuesto un cambio significativo en la gestión turística (Gretzel et al., 2000), en la cual las fronteras entre los procesos offline y online son borrosas tanto desde el punto de vista de la oferta como de la demanda (Ivars et al., 2016). Sin 
Actas del Seminario Internacional Destinos Turísticos Inteligentes:

nuevos horizontes en la investigación y gestión del turismo

Universidad de Alicante, 26 y 27 de octubre de 2017

embargo, el uso inteligente de las mismas en los destinos supone ir más allá de su aplicación y exige cambios estratégicos, en la forma de relacionarse los diferentes agentes, en la mentalidad y la cultura empresarial y en la asimilación de las innovaciones (Ivars, 2013).

Uno de los ámbitos en los que las NTIC ha generado buenos resultados es el referido al turismo cultural. Innovaciones como la realidad aumentada, los códigos QR, los sistemas de recomendación online, las aplicaciones móviles, entre otros, han favorecido el acceso a la oferta cultural y han promocionado el patrimonio de los destinos (SEGITTUR, 2015). En este sentido, cada vez son más comunes la creación de videoguías, las rutas turísticas y las experiencias personalizadas con información geolocalizada, el uso de dispositivos ópticos inteligentes, entre otros (SEGITTUR, 2015). Todos estos ejemplos incluyen uno o varios activos que pueden ser potencialmente protegidos por la propiedad intelectual, con lo cual, para su gestión será necesario considerar el marco legal vigente.

En este contexto, son innegables los beneficios que pueden reportar las NTIC en las diferentes áreas de actividad de un destino. Un análisis de la literatura permite resaltar las siguientes: 1 ) la mejora de la eficiencia interna; 2) el posicionamiento y refuerzo de la imagen de marca; 3) la posibilidad de contar y gestionar un gran volumen de datos; 4) la ampliación de las opciones de distribución de la oferta; y 5) mejora en las interacciones entre los diferentes actores de un destino y a su vez entre diferentes destinos (Cooper et al., 2007; UNWTO, 2008).

Por otra parte, también a nivel empresarial se han destacado las ventajas que ofrecen las NTIC. Entre las más significativas se encuentran: 1) la mejora en las relaciones con los clientes lo cual a su vez contribuye a la calidad, satisfacción y fidelización; 2) el acceso a un mayor número de mercados; 3 ) el reforzamiento de las relaciones con los proveedores; 4 ) el aumento de la eficiencia en las actividades y consecuentemente la reducción de costes operativos; y 5) la posibilidad de planificar los productos y servicios de acuerdo a las necesidades de la demanda (Albacete y Herrera, 2012).

Sin embargo, a pesar de lo previamente comentado, el acceso a las nuevas tecnologías es y estará cada vez más extendido. Por ello, Awad \& Ghaziri (2004) sostienen que la diferenciación y la ventaja competitiva no dependerá tanto del uso de las NTIC sino del planteamiento estratégico que de ellas se haga. Especialmente, del conocimiento que van a generar y de la gestión del mismo. Con lo cual, ya no basta con implementar las NTIC en 
Actas del Seminario Internacional Destinos Turísticos Inteligentes:

nuevos horizontes en la investigación y gestión del turismo

Universidad de Alicante, 26 y 27 de octubre de 2017

los diferentes procesos de las empresas turísticas, sino que resulta esencial gestionar estratégicamente todos los outputs que a través de ellas se pueden crear. Este argumento es perfectamente aplicable a los destinos, sobre todo si consideramos los argumentos previos de Ivars (2013) respecto a que un uso inteligente supone ir más allá de la simple implementación de las NTIC.

Las investigaciones sobre NTIC y turismo se han centrado en tres áreas fundamentales: 1) las dimensiones de consumo y demanda; 2) la innovación tecnológica; y 3) las funciones de la industria (Buhalis \& Law, 2008). Este estudio revisa las tecnologías que han sido identificadas como críticas para el sector turístico, entre las que se encuentran: las tecnologías multimedias, 3D y las tecnologías móviles, entre otras. Por otra parte, Law, et al., (2014) evidencian el rol que desempeñan las redes sociales en la comercialización online y en la toma de decisiones de los clientes. Al tiempo que recomiendan a los directivos la asunción de una actitud proactiva en la que integren los desarrollos tecnológicos a las actividades empresariales diarias y a la misión empresarial. Estas dos importantes revisiones de la literatura permiten constatar la ausencia de estudios sobre las implicaciones que tienen el uso y la implementación de las NTIC respecto a la propiedad intelectual; así como la incidencia de los regímenes de propiedad de estos activos en la gestión turística.

En otro estudio enfocado en las oportunidades y retos que ofrece la aplicación de las tecnologías inalámbricas para el sector turístico, Flouri \& Buhalis (2004) analizan los diferentes agentes que deben cooperar para el desarrollo de aplicaciones turísticas en el destino. En este sentido, argumentan que los proveedores de contenidos son un actor fundamental para la implementación eficaz de las tecnologías en un destino. No obstante, este trabajo no incluye un análisis sobre la regulación legal de los contenidos a utilizar, ni tampoco respecto a las condiciones de uso y acceso a los mismos por los diferentes agentes en el destino.

Si bien hace casi dos décadas Gretzel et al. (2000) identificaban la regulación legal y las cuestiones referidas a la propiedad como algunas de las barreras más importantes para la implementación y la adopción de las tecnologías en los destinos, los temas referidos a la propiedad intelectual han estado prácticamente ausentes en las investigaciones sobre turismo y NTIC. Al mismo tiempo, son puntuales aquellos trabajos en los que se reconoce la importancia del tema para el sector. Vale mencionar a Rodríguez Echavarría et al. (2012) quienes argumentan que los derechos de autor suponen un 
Actas del Seminario Internacional Destinos Turísticos Inteligentes: nuevos horizontes en la investigación y gestión del turismo

Universidad de Alicante, 26 y 27 de octubre de 2017

reto para la sostenibilidad del uso de las nuevas tecnologías en el turismo de patrimonio, especialmente en aquellos países con leyes más conservadoras.

\section{La creación de activos de propiedad intelectual en los destinos turísticos}

La propagación de Internet y las tecnologías digitales, así como la proliferación de las redes sociales han supuesto importantes cambios en la estructura y funciones de la industria turística, pero también en los comportamientos y en los patrones de consumo (Buhalis \& Law, 2008). Desde el punto de vista de la demanda, el viajero ya no es un simple receptor de información, sino que accede a una amplia oferta de información y la comparte a través de las redes sociales (Ivars et al, 2016). Este fenómeno, cada vez más común borra las fronteras entre emisor y receptor y según un informe de SEGITTUR (2015) es considerado como una oportunidad que favorece las posibilidades de complementariedad y cooperación con los usuarios respecto a la generación de contenidos. Esta realidad tiene implicaciones a nivel legal respecto a la autoría y titularidad de aquello que se cocrea con los usuarios y/o entre varios agentes del destino.

Asimismo, las tecnologías digitales y los servicios de geolocalización están impulsando la generación de valor, al tiempo que cambian la forma en la que se crea, intercambia y evalúa la información turística (Law et al., 2011). En un contexto en el que la información es muy valiosa, los datos que generan y acumulan las ciudades son considerados como uno de sus principales activos, por lo cual, la gestión de los mismos es fundamental (Berrone $\&$ Ricart, 2016). En este sentido, existen numerosas iniciativas en las que los diferentes agentes del destino cocrean, intercambian, usan y explotan información tanto entre ellos como con los usuarios. En todos estos casos, hay cuestiones relativas a la propiedad intelectual a considerar en la gestión. Normalmente estas temáticas no se visibilizan en las investigaciones sobre el tema, ni son tenidas en cuenta a la hora de establecer políticas y estrategias sectoriales.

Sin embargo, el aumento de las interacciones virtuales entre usuarios y proveedores de bienes y servicios turísticos (Tseng, et al, 2015) a través de las tecnologías digitales y las redes sociales, no sólo ha devenido en una herramienta para fortalecer las relaciones con los clientes (Anderson \& Srinivasan, 2003), sino que está jugando un rol fundamental en la creación de activos de propiedad intelectual (Mariani, Di Felice, \& Mura, 2016; Shao, et 
Actas del Seminario Internacional Destinos Turísticos Inteligentes:

nuevos horizontes en la investigación y gestión del turismo

Universidad de Alicante, 26 y 27 de octubre de 2017

al, 2016). Vale destacar que las tecnologías móviles, 3D y multimedias son las que integran, cada vez más, los activos de propiedad intelectual en los productos y servicios turísticos. Por lo cual, considerar el marco regulatorio vigente a nivel español y europeo, resulta ineludible en tanto regula las condiciones de distribución y explotación de estos activos.

Un ejemplo ilustrativo sobre la cocreación de activos de propiedad intelectual en el turismo es el proyecto danés Mobile Digital City and Nature Walks, encaminado a desarrollar contenidos y softwares para productos turísticos urbanos y rurales, a través de dispositivos móviles. Este proyecto incluye información georeferenciada sobre los alojamientos, los eventos y las atracciones del destino; además, ponía al alcance de los visitantes contenidos multimedias sobre la mitología vikinga; ofrecía recomendaciones según los perfiles y también ponía en contacto a diferentes usuarios a través del juego online (Nielsen, 2004). Sin embargo, el estudio adolece de un análisis sobre cómo gestionar todos los activos que integran la herramienta teniendo en cuenta la multiplicidad de agentes intervinientes y la legislación vigente.

\section{La gestión de activos de propiedad intelectual}

En la denominada economía del conocimiento, los activos de propiedad intelectual se consideran clave para crear y mantener ventajas competitivas. Para que cumplan este propósito, estos activos necesitan ser gestionados estratégicamente, para lo cual es fundamental que se alineen con la estrategia empresarial (Fisher III \& Oberholzer-Gee, 2013; Lemper, 2012 y Smith \& Hansen, 2002). La literatura sobre gestión de estos activos no define una estrategia única para los mismos, ya que el contexto influye decisivamente en el proceso (Fisher III \& Oberholzer-Gee, 2013), sin embargo, establece una serie de pautas y fases a seguir. A nivel empresarial, las decisiones respecto a estos activos deberán tener en cuenta el rol que tienen los mismos en la actividad central de la empresa. Asimismo, la estrategia para gestionarlos vendrá definida por el papel que juegan en las capacidades centrales de la organización (firm's core capabilities) (Smith \& Hansen, 2002).

Para la gestión de estos activos la auditoría de propiedad intelectual es una herramienta útil y recomendada tanto para las empresas como para las entidades públicas (World Intellectual Property Organization, 2004 y Punnoose \& Shobhana, 2012). Entre las ventajas que se le reconocen se pueden mencionar las siguientes: 1 ) es una herramienta que a las empresas les resulta familiar; y 2) puede utilizarse con diferentes niveles de complejidad 
Actas del Seminario Internacional Destinos Turísticos Inteligentes:

nuevos horizontes en la investigación y gestión del turismo

Universidad de Alicante, 26 y 27 de octubre de 2017

y de este modo adaptarse a los actores y el contexto. Para su utilización se recomienda incluir una fase de identificación de los activos existentes, las formas de protección y explotación, sus fortalezas y riesgos (World Intellectual Property Organization, 2004). Como fase posterior se debe elaborar un plan de propiedad intelectual en el que se incluya una estrategia de gestión y desarrollo de estos activos para un período de tiempo determinado (World Intellectual Property Organization, 2004).

Una de las principales dificultades detectadas del análisis de diferentes estrategias de gestión de los activos de propiedad intelectual es la falta de integración en las funciones legal y de estrategia (Fisher III \& Oberholzer-Gee, 2013). Esta falta de integración se evidencia en problemas de comunicación entre diferentes actores en las fases de investigación, desarrollo y diseño de productos, lo cual genera desencajes con la regulación vigente. Por otra parte, este estudio concluye que la mejor forma de explotar los activos de propiedad intelectual no siempre es eliminando a la competencia a través de los derechos exclusivos que la legislación concede. En este sentido advierten que compartir el valor de estos activos, en ocasiones, puede ser más importante para la sociedad y las empresas. Esta conclusión podría ser igualmente válida para los destinos turísticos inteligentes, en los cuales se promueven sistemas de open data para optimizar los productos y servicios.

Asimismo, existen algunas iniciativas desde el sector privado en las que la apertura de datos por parte de las administraciones públicas se convierte en el elemento clave para la prestación del servicio y el desarrollo de nuevos modelos de negocio. Por ejemplo, a través de la aplicación Moovit es posible planificar trayectos en transporte público entre diferentes ciudades (Berrone \& Ricart, 2016). Otro caso a mencionar es la red CitySDK en la que se ofrecen diferentes servicios urbanos y se promueve la participación activa de las personas a través del envío de información, imágenes, comentarios, entre otros. Todo lo cual contribuye a una mejor gestión de los servicios y eventos en una ciudad (Berrone \& Ricart, 2016). En estos casos acordar previamente las condiciones de acceso y uso de contenidos, bases de datos, software, aplicaciones, entre otros, es fundamental porque en caso contrario rige el marco regulatorio de la propiedad intelectual en el que prima el reconocimiento de la propiedad y por lo tanto, la exclusión al resto de personas y la obligación de pago para acceder a estos activos.

A pesar de las evidentes conexiones entre las NTIC y la gestión de los activos de propiedad intelectual, la literatura especializada en destinos 
Actas del Seminario Internacional Destinos Turísticos Inteligentes:

nuevos horizontes en la investigación y gestión del turismo

Universidad de Alicante, 26 y 27 de octubre de 2017

turísticos inteligentes se ha focalizado en describir el fenómeno a través de casos de estudio (e.g., Boes et al. 2015a, b; Bakici et al. 2013; Wang et al. 2013), analizar desarrollos tecnológicos puntuales (e.g., Huang and Chen 2015; Boes et al. 2015a, b) y abordar los fundamentos teóricos del área (Gretzel, et al., 2015). Sin embargo, queda mucho por avanzar en temas como la coordinación, explotación y condiciones para compartir los datos turísticos en pos de la creación de valor (Gretzel et al., 2015), todo ello a su vez, estrechamente vinculado a la dimensión legal de estos activos, y por lo tanto, al marco regulatorio de la propiedad intelectual. Frente a ese vacío, este estudio persigue ser un primer aporte analítico que conecte estas áreas de estudio.

\section{Metodología}

Este trabajo da continuidad a una investigación previa (Sánchez-Bergara, 2016) enfocada en la gestión de activos de propiedad intelectual en un grupo de empresas turísticas vinculadas a un contexto de innovación. Dada la novedad de la temática, se ha desarrollado a través de un diseño cualitativo para abordar el estudio con una mayor profundidad e incluir elementos del contexto (Patton, 2002). El trabajo se ha realizado en el marco de la tradición interpretativa, concretamente desde una perspectiva constructivista (Guba \& Lincoln, 1994). Es un estudio jurídico-empresarial en el que se incluyen, fundamentalmente, elementos teóricos, empíricos y legales.

En el trabajo empírico efectuado en empresas del sector turístico se optó por un muestreo teórico (Patton, 2002) para garantizar que las empresas que conformaran la muestra utilizaran las redes sociales y las tecnologías digitales en su actividad empresarial. En este sentido, se eligió un contexto que asegurara este perfil de empresas, concretamente un parque científico y tecnológico especializado en la actividad turística en España. Finalmente, se conformó una muestra con 10 organizaciones, pequeñas y medianas empresas y asociaciones sectoriales porque representan al tejido empresarial mayoritario en el turismo, además, dedicadas a diferentes ámbitos de actividad. En la Tabla 1 se resumen sus principales características. 
Actas del Seminario Internacional Destinos Turísticos Inteligentes:

nuevos horizontes en la investigación y gestión del turismo

Universidad de Alicante, 26 y 27 de octubre de 2017

Tabla 1: Características de las organizaciones turísticas participantes

\begin{tabular}{|c|c|c|}
\hline Organización & Fecha de creación & Ámbito de actividad \\
\hline 1 & 2001 & Agencia de viajes \\
\hline 2 & 2011 & Consultoría \\
\hline 3 & 2011 & Comunicación \\
\hline 4 & 2002 & Alojamiento \\
\hline 5 & 2005 & Investigación \\
\hline 6 & 1998 & Náutica \\
\hline 7 & 1999 & Alojamiento \\
\hline 8 & 1979 & Alojamiento y restauración \\
\hline 9 & 2011 & Restauración \\
\hline 10 & 1976 & Alojamiento \\
\hline
\end{tabular}

Fuente: Elaboración propia.

La principal técnica de recolección de datos fue la entrevista, en este caso, se realizaron entrevistas semiestructuradas a los principales directivos de las organizaciones participantes (Berg, 2007). Se desarrollaron en las instalaciones de las organizaciones y tuvieron una duración de entre 45 y 90 minutos. Todas se grabaron y transcribieron íntegramente. Para las entrevistas se elaboró un protocolo fundamentado teóricamente en la literatura de gestión de activos de propiedad intelectual (Punnoose \& Shobhana, 2012 y Smith \& Hansen, 2002) con 33 preguntas organizadas en tres bloques: 1) identificación de los activos de propiedad intelectual utilizados; 2) gestión de estos activos; y 3) marco legal vigente, respecto a las normas legales que regulan el mercado de estos activos.

La obtención de los datos se complementó con fuentes secundarias como: reportes empresariales de las organizaciones participantes extraídos de la base de datos SABI, informes de organismos especializados en gestión de activos de propiedad intelectual, estudios teóricos y empíricos y la legislación sobre derechos de autor vigente a nivel europeo y español. El análisis de estas fuentes permitió validar y triangular datos obtenidos en las entrevistas aportando riqueza y profundidad a la investigación (Denzin, 2012).

El análisis e interpretación de los datos se realizó a través de la combinación de métodos de investigación de las ciencias sociales y de las ciencias 
Actas del Seminario Internacional Destinos Turísticos Inteligentes:

nuevos horizontes en la investigación y gestión del turismo

Universidad de Alicante, 26 y 27 de octubre de 2017

jurídicas. Específicamente, la exégesis jurídica (Vallet de Goytisolo, 1994) y el análisis de contenido cualitativo (Mayring, 2000) o análisis temático (Boyatzis, 1998). La conjugación de estos métodos permitió: el tratamiento de datos de distinta naturaleza y la reducción de códigos deductivos e inductivos (Ryan \& Bernard, 2000). Después de varias fases de reducción y reclasificación surgieron cinco categorías principales a través de las cuales se organizaron los datos. En este trabajo sólo se incluyen dos de ellas: creación y protección porque son las que mayor incidencia tienen desde la perspectiva de los destinos. La categoría creación comprende las áreas de creación, el sujeto creador y la tipología de activos creados. Mientras que la categoría protección se estableció teniendo en cuenta las formas de protección legal y tecnológica disponibles en el contexto español. Estas categorías constituyen la base para contrastar las prácticas seguidas por un grupo de empresas comprometidas con la innovación, las recomendaciones de la literatura sobre gestión de activos de propiedad intelectual y la literatura sobre destinos turísticos inteligentes.

Los resultados que aquí se presentan se construyeron a partir de esta confrontación, verificación de las interpretaciones e inferencia teórica (Seale, 1999).

\section{Resultados}

\subsection{Uso de las NTIC y las redes sociales}

En el grupo de empresas estudiadas la incorporación de las tecnologías digitales en su actividad diaria es un hecho reciente y en buena medida es resultado de un contexto que las presiona a incorporarlas cada vez más en su cotidianidad. En la mayor parte de los casos, estas tecnologías se utilizan en las áreas de gestión interna y comunicación y marketing. En su conjunto se encuentran en diferentes estadios respecto al uso de las tecnologías digitales, pero en ningún caso alcanzan un nivel intensivo, según manifiestan los propios entrevistados.

En relación a las redes sociales se identifican con un rol más activo, si bien también presentan diferentes niveles de uso. Las organizaciones participantes presentan una variada gama de percepciones respecto al rol que tienen las redes sociales para la empresa. Así, se encuentran los dos extremos, desde quien las considera imprescindibles para su actividad hasta un caso que no cuenta con perfil en ninguna red social, pasando por los diferentes 
Actas del Seminario Internacional Destinos Turísticos Inteligentes:

nuevos horizontes en la investigación y gestión del turismo

Universidad de Alicante, 26 y 27 de octubre de 2017

matices entre ambos. En general, consideran que las redes sociales son una herramienta valiosa para la comunicación y el marketing y suelen utilizar sus perfiles para ganar reputación online, fidelizar clientes y mejorar su visibilidad en la web.

Por lo tanto, el contexto de uso de las nuevas tecnologías y las redes sociales dibuja un escenario en el que las organizaciones estudiadas utilizan los activos de propiedad intelectual incrustados en estas tecnologías de manera recurrente, sean o no plenamente conscientes de ello.

\subsection{Creación de activos de propiedad intelectual}

Independientemente del nivel de uso de las tecnologías digitales y las redes sociales, las organizaciones estudiadas crean activos de propiedad intelectual tanto en la realidad física como en la digital. En su conjunto presentan diferencias respecto al nivel de creación, así como en las áreas en las que crean estos activos, la tipología de los mismos y el rol que desempeñan en la actividad central de la empresa.

El área de comunicación y marketing es aquella en la que todas las empresas participantes crean activos de propiedad intelectual. Los activos que se crean con más frecuencia son: textos, imágenes y vídeos. Todos ellos forman parte de una actividad complementaria, ya que están destinados a fortalecer las relaciones con los clientes y generar publicidad online, fortaleciendo así la reputación en el ámbito digital. Estos activos son creados por: 1) personas con un contrato laboral con la empresa participante; 2) terceras personas contratadas ocasionalmente; y 3) los usuarios.

Por otra parte, en el área de productos y servicios son pocas las organizaciones que crean activos de propiedad intelectual, sin embargo, aquellas que lo hacen generan activos de distinta naturaleza a los previamente mencionados y lo hacen como parte de su actividad principal. Específicamente, aplicaciones para móviles, bases de datos, programas informáticos y obras multimedias. Estos activos son creados por: los trabajadores; o cocreados junto a usuarios o colaboradores tecnológicos.

Tanto las organizaciones que crean activos de propiedad intelectual como parte de su actividad central como aquellas que lo hacen como actividad complementaria coinciden en la corta vida de estos activos. Los directivos entrevistados coinciden en que estos bienes tiene una vida útil breve, ya sea por la velocidad con la que evolucionan las tecnologías y los contenidos 
Actas del Seminario Internacional Destinos Turísticos Inteligentes:

nuevos horizontes en la investigación y gestión del turismo

Universidad de Alicante, 26 y 27 de octubre de 2017

en las redes, como porque la funcionalidad de los mismos exige de renovaciones periódicas.

En síntesis, todas las empresas estudiadas crean activos de propiedad intelectual en su cotidianidad, si bien, en la mayoría de los casos, estos activos juegan un rol subordinado respecto a la actividad central de la empresa. De manera unánime son considerados activos con una rápida obsolescencia.

\subsection{Protección de activos de propiedad intelectual}

Respecto a la categoría de protección, en general los datos evidencian que las organizaciones estudiadas no conocen ni entienden qué significa 'proteger' los activos de propiedad intelectual. En su gran mayoría no utilizan ni mecanismos legales ni tecnológicos para proteger estos activos. Sólo en casos puntuales se encuentran protegidos y precisamente se corresponden a las organizaciones que crean estos activos como parte de su actividad principal.

Resulta interesante destacar que sólo una de las organizaciones participantes cuenta con mecanismos de protección legal y tecnológica, así como en determinados casos opta por no proteger estos activos. Es decir, adopta tres posturas diferentes en función del tipo de activo y la explotación que de ellos hará. La protección legal la utiliza cuando le interesa excluir estos activos de la competencia. Mientras que excluye la protección en aquellos activos que destina a ganar reputación y mejorar su imagen en las redes sociales.

Por otra parte, otra de las organizaciones que sí ha realizado un análisis previo sobre cuáles eran las formas óptimas de protección de sus activos de propiedad intelectual, ha optado por protegerlos a través de mecanismos tecnológicos. La decisión se ha fundamentado, por una parte, en el tipo de explotación que harían de los activos y por otra, porque en la evaluación realizada concluyeron que era un mecanismo de protección más eficaz que el jurídico.

\section{Discusión y conclusiones}

El desarrollo y buen funcionamiento de los destinos turísticos inteligentes exige que las NTIC estén presentes de manera extendida entre todos los agentes del destino. También es necesario que la utilización que de ellas se haga promueva un uso eficiente de los recursos y facilite el acceso a 
Actas del Seminario Internacional Destinos Turísticos Inteligentes:

nuevos horizontes en la investigación y gestión del turismo

Universidad de Alicante, 26 y 27 de octubre de 2017

productos y servicios adaptados a la demanda. El análisis de los resultados obtenidos sugiere que la inclusión de las NTIC no es homogénea entre las empresas y los niveles de incorporación de las mismas varían de manera significativa. Si tenemos en cuenta que las PYMEs son un eslabón fundamental en el sector, una de las primeras barreras a superar para formar parte de un destino turístico inteligente será la incorporación y uso estratégicos de las NTIC por el entramado empresarial del destino. En este sentido, tanto las administraciones públicas como las asociaciones sectoriales deberán jugar un rol determinante en el apoyo, formación y acceso a las NTIC por parte de las PYMEs.

Del análisis de los resultados se constata que el uso de las NTIC y las redes sociales favorece la creación y cocreación de activos de propiedad intelectual como parte de la cotidianidad de las organizaciones del sector turístico. Esta conclusión, es coherente con las conclusiones de Punnoose \& Shobhana (2012) respecto a que las organizaciones crean activos de propiedad intelectual sin importar el sector industrial al que pertenezcan. A nivel de DTI esta realidad estará mucho más presente debido al rol transversal que tienen las NTIC y a la importancia de la innovación. Sin embargo, la ventaja competitiva tal y como sostienen Awad \& Ghaziri (2004) el factor diferencial vendrá dado por la creación y gestión estratégica de activos de propiedad intelectual y no tanto por el uso de las NTIC porque las mismas estarán cada vez más democratizadas.

Estos elementos suponen retos respecto al régimen de utilización de activos que pueden ser creados y utilizados por multiplicidad de agentes, en ocasiones además, con diferentes nacionalidades, lo cual añade un elemento de complejidad respecto a la presencia de un elemento extranjero en las relaciones que se entablan. En esta línea, lo primero a definir es el régimen bajo el que estarán los activos de propiedad intelectual. Siempre teniendo en cuenta que rige la legislación vigente salvo expreso acuerdo entre todos los agentes involucrados. En caso de que no haya pronunciamiento en este sentido, regirán las normas legales de la materia.

La constatación de que las empresas turísticas estudiadas crean y cocrean activos de propiedad intelectual -imágenes, textos, vídeos, aplicaciones para móviles, programas de ordenador, bases de datos- como parte de su actividad diaria, evidencia la necesidad de considerar cuestiones referidas a la gestión de estos activos, de las cuales tampoco estarán exentos los DTI. Por una parte, la creación a partir de aportaciones individuales de varias 
Actas del Seminario Internacional Destinos Turísticos Inteligentes:

nuevos horizontes en la investigación y gestión del turismo

Universidad de Alicante, 26 y 27 de octubre de 2017

personas y/o empresas añade complejidad respecto a la delimitación de la autoría y titularidad de estos bienes, lo cual a su vez tendrá efectos directos en la explotación de los mismos. Por otra parte, el encargo de este tipo de activos a terceras personas y/o empresas exige una clara delimitación de la titularidad y condiciones de explotación de los mismos en los acuerdos previos, debido a que la legislación y la jurisprudencia españolas no resultan claras ni uniformes en este punto.

Respecto a las modalidades de protección existe poco conocimiento sobre el tema en las organizaciones estudiadas, en parte debido a la falta de cultura empresarial respecto a gestionar los activos de propiedad intelectual, así como el tamaño y juventud de la mayor parte de ellas. Es por ello que la gran mayoría no ha tomado decisiones estratégicas en este ámbito. Con independencia de que se opte por excluir tanto la protección legal como la tecnológica, en la medida en que los activos de propiedad intelectual son cada vez más valiosos, es recomendable que tanto empresas como los destinos tomen decisiones al respecto teniendo en cuenta: 1) la tipología del bien; 2) el fin que con él se persigue; y 3) las formas de explotación a las que previsiblemente estará sujeto. La literatura evidencia que en determinados sectores la protección legal se considera innecesaria y son capaces de desarrollar su creatividad e innovación al margen del régimen de la propiedad intelectual (e.g. Raustiala \& Christopher, 2006; Buccafusco, 2007; Fry, 2010). Estos ejemplos podrían ser un referente para los DTI porque son industrias en las que la exclusividad que concede la protección legal resulta contraproducente (Rosenblatt, 2013), situación que presenta ciertas semejanzas con el funcionamiento y gestión de los DTI.

Desde una visión general de gestión, los resultados evidencian que las empresas estudiadas tienen serias limitaciones para incorporar los activos de propiedad intelectual en la estrategia empresarial. Entre las principales barreras pueden mencionarse la falta de experiencia en la gestión de estos activos, los limitados recursos humanos con que cuentan y la falta de experticia de los mismos en este ámbito. Esta situación restringe la creación de valor y de ventajas competitivas a partir de estos activos. Considerando estos hechos, así como que probablemente otras empresas con un perfil similar se encuentren en una situación similar, se hace necesario que los gestores y directivos se conciencien en el tema y favorezcan la formación de sus subordinados. Esta conclusión es trasladable a los distintos entes de un DTI, ya que se verán en la necesidad de gestionar no sólo sus propios activos de 
Actas del Seminario Internacional Destinos Turísticos Inteligentes:

nuevos horizontes en la investigación y gestión del turismo

Universidad de Alicante, 26 y 27 de octubre de 2017

propiedad intelectual, sino también los de otros agentes con los que interactúan. En este sentido, sería recomendable que las asociaciones sectoriales, las administraciones públicas y otros entes de gobernanza del destino ofrezcan asesoría y acompañamiento en la temática, especialmente a las PYMEs.

Finalmente, vale destacar que una gestión inteligente de los activos de propiedad intelectual supondrá dejar de lado las actuaciones reactivas e incluir estos activos dentro de la estrategia empresarial y la estrategia del destino. Considerando que este es un primer estudio cualitativo con una muestra teórica reducida sería conveniente profundizar en la temática con nuevos criterios muestrales que permitan identificar los elementos diferenciadores en la gestión de estos activos entre empresas y destinos. Asimismo, sería conveniente analizar los efectos de utilizar diferentes modalidades de protección y regímenes de propiedad para estos activos en la gestión de los DTI. De este modo, los diferentes agentes contarán con evidencias teóricas y empíricas para promover políticas e incidir en el marco regulatorio vigente tanto a nivel español como europeo.

\section{Referencias}

Albacete, C. A. y Herrera, M. (2012). Las tecnologías de la información y la comunicación en el sector turístico: Una aproximación al uso de las redes sociales. Madrid: Centro de Estudios Ramón Areces.

Anderson, R. E., \& Srinivasan, S. S. (2003). E-satisfaction and e-loyalty: A contingency framework. Psychology and Marketing, 20(2), 123-138.

Awad, E. M., \& Ghaziri, H. M. (2004). Knowledge Management. New Jersey: Pearson Education Inc.

Berrone, P., \& Ricart, J. E. (2016). La gobernanza inteligente, clave para las «smart cities.» Harvard Deusto Business Review, 254, 14-21.

Bakici, T., Almirall, E., \& Wareham, J. (2013). A smart city initiative: the case of Barcelona. Journal of the Knowledge Economy, 4(2), 135-148.

Berg, B. L. (2007). Qualitative research methods for the social sciences. Boston: Pearson Education Inc.

Boes, K., Borde, L., \& Egger, R. (2015a). The Acceptance of NFC Smart Posters in Tourism. In I. Tussyadiah \& A. Inversini (Eds.), Information and Communication Technologies in Tourism 2015 (pp. 435-448). Heidelberg: Springer.

Boes, K., Buhalis, D., \& Inversini, A. (2015b). Conceptualising Smart Tourism Destination Dimensions. In I. Tussyadiah \& A. Inversini (Eds.), Information and Communication Technologies in Tourism 2015 (pp. 391-403). Heidelberg: Springer. 
Actas del Seminario Internacional Destinos Turísticos Inteligentes:

nuevos horizontes en la investigación y gestión del turismo

Universidad de Alicante, 26 y 27 de octubre de 2017

Buccafusco, C. J. (2007). On the legal consequences of sauces: should Thomas Keller's recipes be per se copyrightable? Cardozo Arts \& Entertainment Law Journal, 24(3), 1121-1156.

Buhalis, D. (2003). E-tourism: Information technology for strategic tourism management. Londres: Pearson (Financial Times / Prentice-Hall).

Buhalis, D., \& Law, R. (2008). Progress in information technology and tourism management: 20 years on and 10 years after the internet-The state of eTourism research. Tourism management, 29, 609-623.

Denzin, N. K. (2012). Triangulation 2.0. Journal of Mixed Methods Research, 6(2), 80-88.

Fisher III, W. W., \& Oberholzer-Gee, F. (2013). Strategic Management of Intellectual Property: An Integrated Approach. California Management Review, 55(4), 157183.

Flouri, E., \& Buhalis, D. (2004). Wireless technologies for tourism destinations. In A. J. Frew (Ed.), Information and communication technologies in tourism 2004 (pp. 27-38). New York: Springer Wien.

Fry, B. (2010). Why Typefaces Proliferate without Copyright Protection. Journal on Telecommunications and High Technology Law, 8(2), 425-490.

Garon, J. (2012). The Heart of the Deal: Intellectual Property Aspects in the Law and Business of Entertainment. Journal of Intellectual Property Rights, 17(September), 443-453.

Ghafele, R., \& Santagata, W. (2006). Cultural Tourism and Collective Trademarks : The Case of Byblos and Saida, Lebanon. Turin.

Gretzel, U.; Yuan, Y. y Fesenmaier, R. (2000). «Preparing for the New Economy: Advertising Strategies and Change in Destination Marketing Organizations». Journal of Travel Research, 39, 146-156.

Gretzel, U., Sigala, M., Xiang, Z., \& Koo, C. (2015). Smart tourism: foundations and developments. Electronic Markets, 25(3), 179-188. https://doi.org/10.1007/ s12525-015-0196-8.

Ide, A. (2008). Regional Developments by Making Intellectual Property Public Domain. Journal of Travel \& Tourism Marketing, 24(2-3), 239-242. https://doi. org/10.1080/10548400802092908.

Ivars, J. A. (2013). «Destinos turísticos inteligentes». En: Solsona, J. y Pardellas, X. (dir.). La actividad turística española en 2011. Madrid: Ramón Areces. Asociación Española de Expertos Científicos en Turismo, 473-476.

Ivars Baidal, J. A., Solsona Monzonís, F. J., \& Giner Sánchez, D. (2016). Gestión turística y tecnologías de la información y la comunicación (TIC): El nuevo enfoque de los destinos inteligentes*. Documents d'Anàlisi Geogràfica, 62(2), 327-346. https://dx.doi.org/10.5565/rev/dag.285. 
Actas del Seminario Internacional Destinos Turísticos Inteligentes:

nuevos horizontes en la investigación y gestión del turismo

Universidad de Alicante, 26 y 27 de octubre de 2017

Kankanala, K. C. (2012). Business Value from Intellectual Property. Journal for InteIlectual Property Rights, 17(40), 369-373.

Law, R., Buhalis, D., \& Cobanoglu, C. (2014). Progress on information and communication technologies in hospitality and tourism. International Journal of Contemporary Hospitality Management, 26(5), 727-750.

Law, R., Fuchs, M., \& Ricci, F. (2011). Information and communication technologies in tourism 2011. Vienna, Austria: Springer Verlag.

Lemper, T. A. (2012). The critical role of timing in managing intellectual property. Business Horizons, 55(4), 339-347.

Mariani, M. M., Di Felice, M., \& Mura, M. (2016). Facebook as a destination marketing tool: Evidence from Italian regional Destination Management Organizations. Tourism Management, 54, 321-343.

Miralbell, O. y Sivera, S. (2008). «New innovation networks in destinations 2.0». XVI Simposi Internacional de Turisme i Oci. Barcelona: ESADE.

Mukundan, R., \& Jain, K. (2012). Enhancing a Firm 's Strategic Intellectual Property Management System - The Role of Patent Quality. Journal of Intellectual Property Rights, 17(September), 385-389.

Nielsen, L. B. (2004). Post Disney experience paradigm? In Proceedings of the 6th international conference on Electronic commerce - ICEC'04 (p. 657). New York, New York, USA: ACM Press.

Ohashi, Y., Ohashi, K., Meskanen, P., Hummelin, N., Kato, F., \& Kynäslahti, H. (2012). What children and youth told about their home city in digital stories in «C my city!» Digital Creativity, 23(2), 126-135. https://doi.org/10.1080/14626268.20 12.709942.

Punnoose, S., \& Shobhana, V. (2012). The Intellectual Property Audit. Journal of Intellectual Property Rights, 17(September), 417-424.

Raustiala, K., \& Christopher, S. (2006). The piracy paradox: innovation and intellectual property in fashion design. Virginia Law Review, 92(8), 1687-1777.

Rodríguez Echavarría, K., Kaminski, J., \& Arnold, D. (2012). 3D Heritage on Mobile Devices: Scenarios and Opportunities. In M. Ioannides, D. Fritsch, J. Leissner, R. Davies, F. Remondino, \& R. Caffo (Eds.), Progress in Cultural Heritage Preservation (pp. 149-158). Berlin, Heidelberg: Springer Berlin Heidelberg. https://doi. org/10.1007/978-3-642-34234-9.

Rosenblatt, E. L. (2013). Intellectual property's negative space: beyond the utilitarian. Florida State University Law Review, 40(3), 441-486.

Russo, A. P. (2013). Branding brazilian slums through freeware cultural production the case of Rio de Janeiro. In F. M. Go \& R. Govers (Eds.), International Place Branding Yearbook. Managing smart growth \& Sustainability. 
Actas del Seminario Internacional Destinos Turísticos Inteligentes:

nuevos horizontes en la investigación y gestión del turismo

Universidad de Alicante, 26 y 27 de octubre de 2017

Russo, A. P., \& Segre, G. (2009). Destination Models and Property Regimes. Annals of Tourism Research, 36(4), 587-606. https://doi.org/10.1016/j. annals.2009.04.002.

Sánchez-Bergara, S. (2016). Derechos de autor y conexos y nuevas tecnologías en el ocio y el turismo: reconfiguración legal, construcción de mercado y valor empresarial. TDX (Tesis Doctorals en Xarxa). Universitat Rovira i Virgili. Retrieved from http://www.tdx.cat/handle/10803/398611.

Santagata, W. (2002). Cultural Districts, Property Rights and Sustainable Economic Growth. International Journal of Urban and Regional Research, 26(1), 9-23. https://doi.org/10.1111/1468-2427.00360.

Santagata, W. (2006). Chapter 31 Cultural Districts and Their Role in Developed and Developing Countries. Handbook of the Economics of Art and Culture, 1, 11011119. https://doi.org/10.1016/S1574-0676(06)01031-3.

Seale, C. (1999). The Quality of Qualitative Research. Sage Publications.

SEGITTUR. (2015). Informe destinos turísticos inteligentes: construyendo el futuro. Segittur. Madrid.

Segre, G., \& Russo, A. P. (2005). Collective property rights for glass manufacturing in Murano: where culture makes or breaks local economic development (Working Paper Series International Centre for Research on the Economics of Culture, Institutions and Creativity No. 5). Turin.

Shao, J., Li, X., Morrison, A. M., \& Wu, B. (2016). Social media micro-film marketing by Chinese destinations: The case of Shaoxing. Tourism Management, 54, 439451.

Sheldon, P. J. (1997). Tourism Information Technology. Wallingdorf: CABI.

Smith, M., \& Hansen, F. (2002). Managing intellectual property: a strategic point of view. Journal of Intellectual Capital, 3(4), 366-374. https://doi. org/10.1108/14691930210448305.

Spinello, R. a. (2007). Intellectual property rights. Library Hi Tech, 25(1), 12-22. https://doi.org/10.1108/07378830710735821.

UNCTAD. (2010). Economía Creativa: Una opción factible de desarrollo.

Wang, D., Li, X. R., \& Li, Y. (2013). China's Bsmart tourism destination initiative: a taste of the service-dominant logic. Journal of Destination Marketing and Management, 2(2), 59-61.

William, E. (2010). Ecosistema del turismo red: Modelo de la abundancia e innovación en las Islas Canarias. Universidad de Las Palmas de Gran Canaria. Tesis doctoral.

World Intellectual Property Organization. (2004). IP Asset Development and Management: A Key Strategy for Economic Growth. Ginebra: World Intellectual Property Organization. 